\title{
Colour Image Watermarking using Bi-Orthogonal Wavelet Transform
}

\author{
K. Ramani \\ Professor, Dept. of IT \\ SVEC, Tirupati \\ A. P, India
}

\author{
E.V.Prasad \\ Professor, Dept. of CSE \\ JNT University, \\ Kakinada, A. P, India
}

\author{
V. Lokanadham Naidu \\ Asst. Professor (SL), Dept. of IT \\ SVEC, Tirupati, A. P, India
}

\author{
D. Ganesh \\ Asst. Professor \\ Dept. of IT, SVEC, \\ Tirupati, A. P, India
}

\begin{abstract}
The proposed method provides copyright protection for digital images. This watermarking algorithm embeds a logo invisibly into the colour biomedical image which is decomposed using biorthogonal wavelet transform using two pn-sequences. Biorthogonal wavelets have the property of perfect reconstruction and smoothness. The colour cover image is decomposed into R, G, $\mathrm{B}$ channels and blue channel is selected for watermarking. The blue channel is decomposed into n-levels using bi-orthogonal wavelet transform and mid-frequency bands LH and HL are selected for embedding. The prosed method is verified for different cover images and with different watermaks. The robustness of this algorithm is tested against various types of image processing operations and geometric attacks such as Salt and pepper noise, Gaussian noise, Poisson noise, Compression, Rotation, Scaling and Cropping.
\end{abstract}

\section{General Terms}

Image Processing, Watermarking, Information Security.

\section{Keywords}

Bi-orthogonal wavelets, bio-medical images, digital watermarking, Peak Signal to Noise ratio, Normalized Cross Correlation, Geometrical attacks and image processing operations.

\section{INTRODUCTION}

As the audio, video, and multimedia products were rapidly distributed over the fast communication systems such as Internet and satellite. The strategies of resolving copyright ownership and verifying the originality of digital contents are urgently requested. As a new solution for content protection, digital watermarking, is drawing considerable attention and becomes an active research field. This paper concentrates on the verification of copyright ownership of still images by a watermarking scheme, with a watermark being regarded as a set of random signals which are embedded into an image to protect the copyright of the owner.

The objective of the watermarking is to hide data for digital right management and copyright protection of the digital image, video or audio that holds the secret watermark. There are a lot of the watermarking applications such as: broadcast monitoring in which the watermark is embedded in the advertising spot by the advertisers. In this way, we will know if another station pirated the advertisement that has their watermark. Copy control, while some companies allow recording TV broadcast, they don't allow recording another copy of the broadcast and that is accomplished by adding a fragile watermark that says (copy allowed) which will not appear if a video recorder is used. Another application is Content authentication which is done by adding a watermark to digital works, photographs, surveillance camera videos as well as important scanned documents [1].
Algorithm robustness is important from different point of views. In copyright protection an algorithm should be robust against all kinds of removal attacks including common signal processing distortions which an image encounters during transmission, and malicious removing attacks. Algorithm robustness is important from different point of views. In copyright protection an algorithm should be robust against all kinds of removal attacks including common signal processing distortions which an image encounters during transmission, and malicious removing attacks. In this regard, many different types of approaches have been reported in the literature. Some of them simply work in spatial domain. At present, these algorithms are not in wide use; since their general robustness does not seem adequate. Some other algorithms use different types of 2-D image transforms to embed their watermark signal more robustly. Amongst these the discrete cosine transform-based and wavelet-based algorithms seem to be more promising. The wavelet-based algorithms have shown to be much more robust and perform greater perceptual invisibility than others [2, 3, 4]. Some wavelet-based algorithm have been designed to embed the watermark signal into the lower level subbands. Most of them do not consider the fact that embedding watermark in higher-level subbands makes the algorithm much more robust due to quality degradation. In this paper, we present a robust multiresolution image watermarking method with application to copyright protection of images. This method explicitly exploits the human visual system to guarantee that the embedded watermark is imperceptible. The proposed algorithm saves the image quality in spit of changing all wavelet coefficients of original image.

We have described other related work in Section 2, our proposed watermark embedding and detection algorithm in Section 3. The experimental results are given in Section 4, followed by conclusion in Section 5.

\section{RELATED WORK}

Cox [5] first proposed an algorithm that inserts watermarks into the spectral components of the image using techniques analogous to spread-spectrum communication. The algorithm is to place the watermark into a set of frequency components that are perceptually significant. It has been shown that the watermarks are very hard to detect because they consist of relatively weak noise signals. In addition, placing the watermarks in the frequency domain would spread them over all pixels, which increases the robustness, and reliability against an unintentional or intentional attack $[6,7]$. T. Tsui et. al.[8] provided a method for embedding a watermark into colour cover image using Fourier Transform.

N. Ahmidi et. al [9] presented a watermarking algorithm for embedding a logo into colour image using DCT. Roland Kwitt et.al [10] introduced the one of the frequency transform domain 
watermarking schemes. They used discrete wavelet transform (DWT) technique to embed the signal bits in the image. They also reported an efficient DWT domain watermarking technique that could resist some of the major attacks.

\section{PROPOSED WATERMARKING \\ METHOD}

A. Embedding Process

1. Read color image, I(x, y).

2. Separate the channels R, G, B of I(x,y).

3. Decompose each channel using 2nd-level Bi- orthogonal 1.1 wavelet transform.

4. After decomposition, each subband size will be:

Where,

$$
(\mathrm{X} \times \mathrm{Y}) / 2^{\mathrm{k}} \times 2^{\mathrm{k}}
$$

$$
\mathrm{X} \times \mathrm{Y} \text { is size of cover image }
$$

$\mathrm{k}=$ level of decomposition $(\mathrm{k}=2)$

5. Read watermark bitmap image of size $60 \times 60$ and convert it into $1-\mathrm{D}$ vector.

6. To generate watermark sequence (w), use two pseudorandom noise signals $(\mathrm{PN})$.

PN_0: used for embedding watermark bit 0 and

PN_1: used for embedding watermark bit 1

7. Two subbands (mid-band) are selected, $\mathrm{HL}_{2}$ and $\mathrm{LH}_{2}$ for embedding keeping Human visual system (HVS) characteristics intact.

8. By using additive water marking techniques construct the image as:

$$
\begin{aligned}
& \mathrm{LH}_{2}{ }^{\prime}=\mathrm{LH}_{2}+\alpha * \mathrm{PN}{ }_{-} 0 \\
& \mathrm{HL}_{2}{ }^{\prime}=\mathrm{HL}_{2}+\alpha * \mathrm{PN}_{-} 1 \ldots \ldots \ldots . . . . \\
& \mathrm{LH}_{2}{ }^{\prime} \text { and } \mathrm{HL}_{2}{ }^{\prime} \rightarrow \text { watermarked subbands } \\
& \mathrm{LH}_{2} \text { and } \mathrm{HL}_{2} \rightarrow \text { original subbands } \\
& \alpha \rightarrow \text { embedding strength }
\end{aligned}
$$

9. Using inverse bi-orthogonal wavelet transform reconstruct the watermarked image, I'(X, Y).

10. Peak Signal to Noise Ration is calculated between original cover image and watermarked image using the following formula:

$\operatorname{PSNR}(\mathrm{dB})=10 \log _{10}\left(255^{2} / \mathrm{MSE}\right)$

\section{B. Extraction Process}

1. Read the watermarked image I' (X, Y).

2. Separate R,G,B Channels.

3. Decompose each channel using 2-level bi-orthogonal 1.1 wavelet transform.

4. Select the subbands $\mathrm{HL}_{2}{ }^{\prime}$ and $\mathrm{LH}_{2}{ }^{\prime}$.

5. Generate PN_1 and PN_0 signal similarly to embedding process and select the threshold value.

6. Extract the watermark from each subband.

7. Normalized cross correlation is calculated between original and extracted watermarks, using Eqn. (4)

$$
\rho=\sum_{i=1}^{N} \varpi_{i} \omega_{i}^{\prime} / \sqrt{\sum_{i=1}^{N} \varpi_{i}} \cdot \sqrt{\sum_{i=1}^{N^{*}} \varpi_{i}^{\prime}}
$$

\section{EXPERIMENTAL RESULTS}

Football.bmp colour image of size 256X256 is selected as a cover image and skeleton.bmp grey scale image of size 60X60 is chosen as a watermark. Blue channel of the cover image is selected for embedding process, as blue channel is more resistance to changes compared to red and green channels. Figure.1. shows the original image, separated blue channel, output of 1-level decomposed blue channel using bior1.1 wavelet transform, watermarked blue channel, watermarked football.bmp, original watermark, skeleton.bmp are shown respectively. Figure.2. shows the watermarked image and extracted watermark at the receiver side.

The performance of the proposed method for image processing operations such as Gaussian noise, Poisson noise, Salt and pepper noise, JPEG compression and geometrical attacks such as Scaling, Rotation and Cropping is studied and experimentally obtained results are shown in Table 1 for football.bmp. Similarly, the performance of the algorithm for X-ray.bmp colour bio-medical image at second level bior 1.1 is for the watermark skeleton.bmp is studied and the experimental results are shown in Table 2.

The PSNR and Cross correlation values obtained for different cover images for different types of image processing and geometrical attacks is shown in Table 3.Embedding area depends on level of decomposition, hence the algorithm is also verified with different watermark sizes, the results obtained are shown in Table 4.

The algorithm is also verified with haar wavelet transform and experimentally obtained results are compared with bior 1.1 transform results, as shown in Figure. 3. to Figure. 8. There is no much difference in PSNR values obtained by both methods, only Normalized Cross correlation values varying.

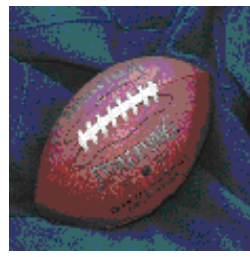

(a)

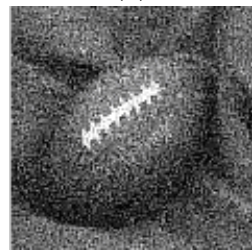

(d)

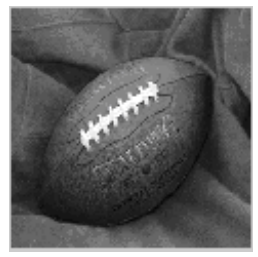

(b)

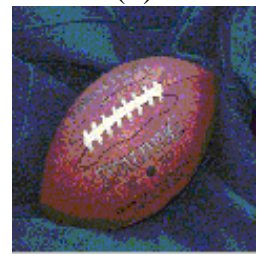

(e)

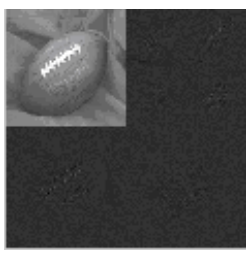

(c)

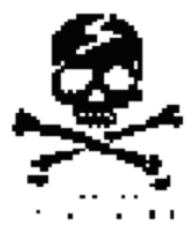

(f)
Figure 1. Embedding watermark at sender side (a) Original cover image (b) Blue channel image (c) DWT $1^{\text {st }}$ level decomposition (d) Watermarked blue channel image (e) Watermarked image (f) Original watermark 


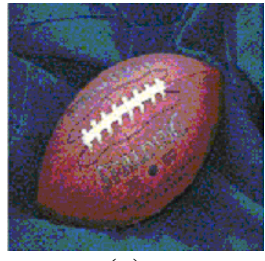

(a)

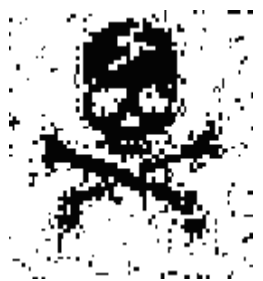

(b)
Figure 2. Extraction of watermark at receiver side (a) Watermarked image (b) Extracted watermark

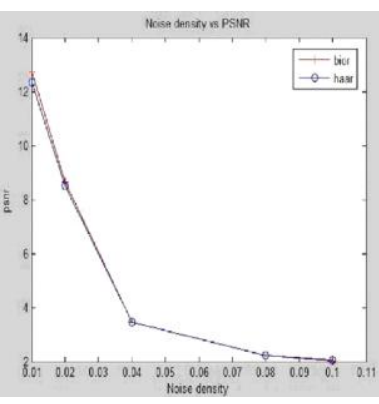

(a)

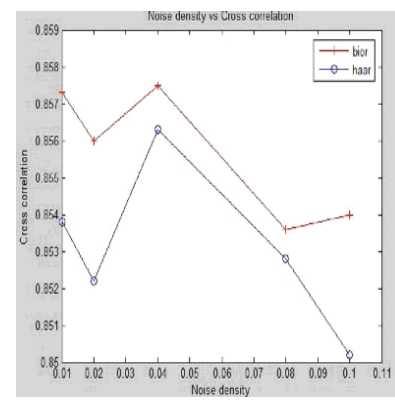

(b)
Figure 3. (a) Noise density Vs PSNR (b) Noise density Vs Normalized Cross correlation using Haar and bior1.1, with Gaussian noise operation on watermarked image.

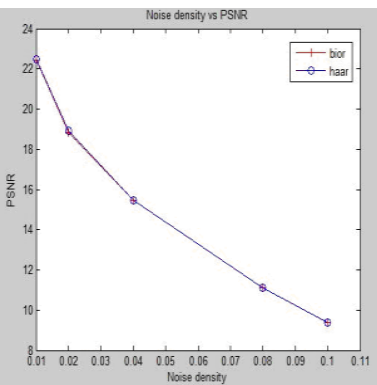

(a)

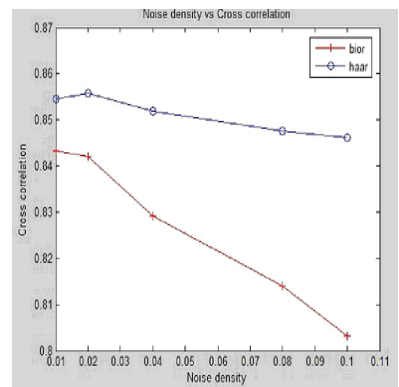

(b)
Figure 4. (a) Noise density Vs PSNR (b) Noise density Vs Normalized Cross correlation using Haar bior1.1, with Salt and Pepper noise operation on watermarked image.

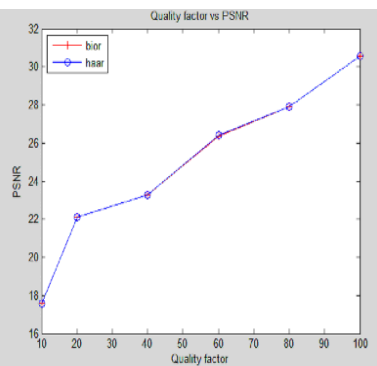

(a)

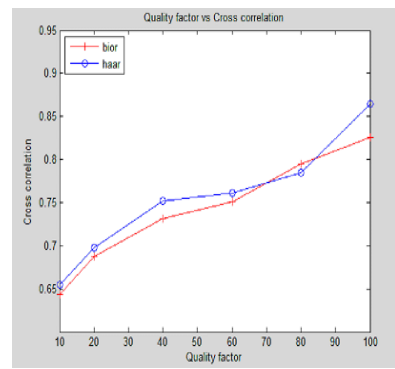

(b)
Figure 5. (a) Quality factor Vs PSNR (b) Quality factor Vs Normalized Cross correlation using Haar and bior1.1 with JEPG compression attack on watermarked image.

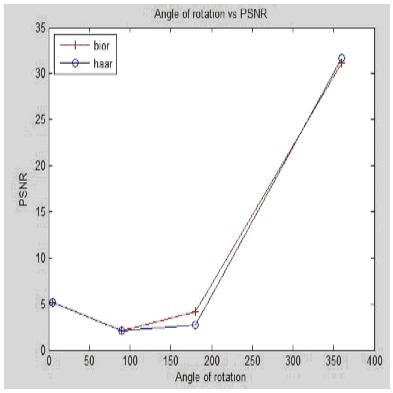

(a)

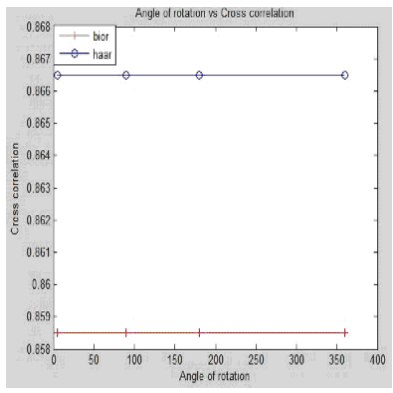

(b)
Figure 6. (a) Angle of rotation Vs PSNR (b) Angle of rotation Vs Normalized Cross correlation using Haar and bior1.1 with rotation attack on watermarked image.

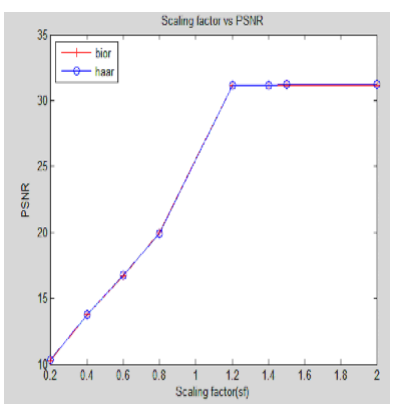

(a)

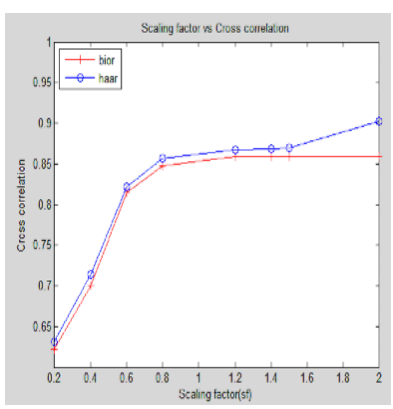

(b)
Figure 7. (a) Scaling factor Vs PSNR (b) Scaling factor Vs Normalized Cross correlation using Haar and bior1.1 with scaling attack on watermarked image.

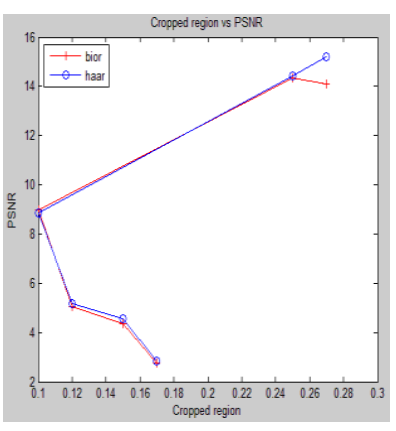

(a)

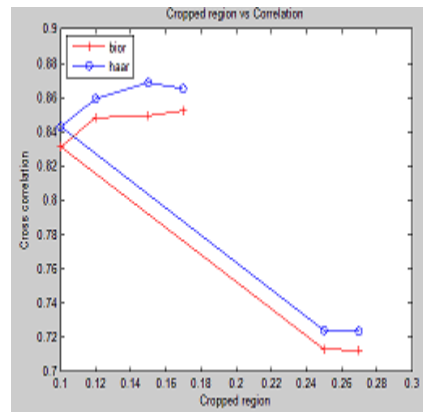

(b)
Figure 8. (a) Cropped region Vs PSNR (b) Cropped region Vs Normalized Cross correlation using Haar and bior1.1 with Cropping attack on watermarked image. 
Table 1. Performance of the proposed algorithm against various types of attacks, at first level decomposition using bior 1.1

\begin{tabular}{|c|c|c|}
\hline $\begin{array}{l}\text { Attack/Image } \\
\text { Processing } \\
\text { Operation } \\
\end{array}$ & $\begin{array}{l}\text { Watermarked image } \\
\text { after attack/ Image } \\
\text { Processing Operation }\end{array}$ & $\begin{array}{c}\text { Extracted } \\
\text { Watermark }\end{array}$ \\
\hline $\begin{array}{c}\text { Gaussian noise } \\
\text { (noise } \\
\text { density }=0.04)\end{array}$ & & 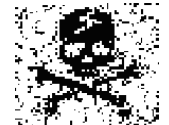 \\
\hline Poissian noise & & \\
\hline $\begin{array}{l}\text { Salt and pepper } \\
\text { noise } \\
\text { (noise } \\
\text { density }=0.04 \text { ) }\end{array}$ & & \\
\hline $\begin{array}{c}\text { JPEG } \\
\text { compression } \\
\text { (compression } \\
\text { factor=40) }\end{array}$ & & 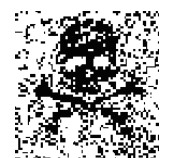 \\
\hline $\begin{array}{c}\text { Cropping } \\
\text { (cropping range } \\
{[1010200} \\
200] \text { ) }\end{array}$ & & 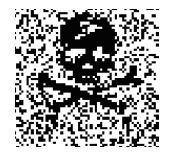 \\
\hline $\begin{array}{l}\text { Rotation attack } \\
\quad(\text { angle of } \\
\left.\text { rotation }=140^{\circ}\right)\end{array}$ & & कen \\
\hline $\begin{array}{l}\text { Scaling (noise } \\
\text { density }=0.6 \text { ) }\end{array}$ & & 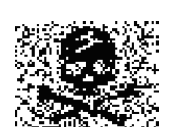 \\
\hline
\end{tabular}

Table 2. Performance of the proposed algorithm against various types of attacks, at second level decomposition using bior 1.1

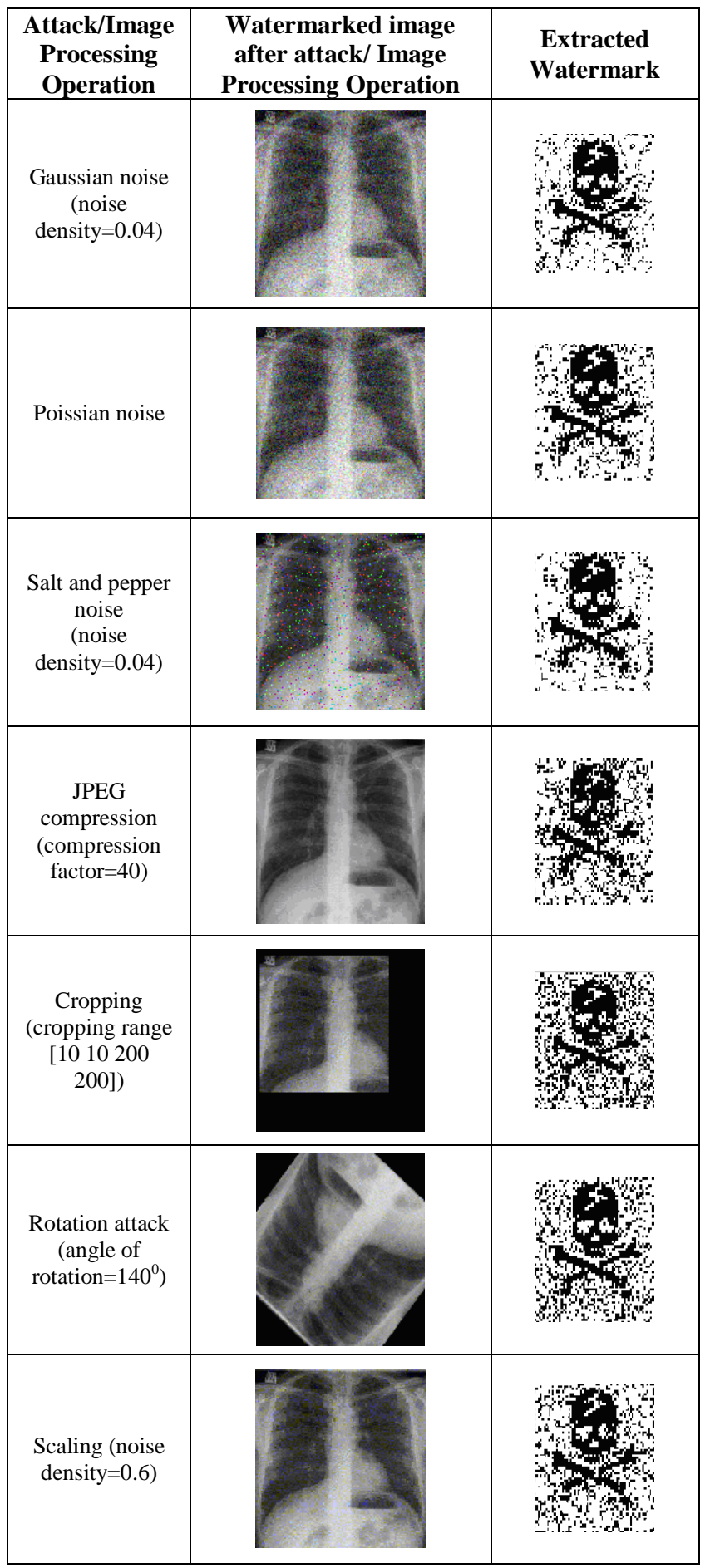


Table 3. Comparison of PSNR and Normalized Cross correlation values for different cover images

\begin{tabular}{|c|c|c|c|c|}
\hline K=5 & \multicolumn{2}{|c|}{ FOOTBALL IMAGE } & \multicolumn{2}{|c|}{$\begin{array}{c}\text { MEDICAL } \\
\text { IMAGE (X-RAY) }\end{array}$} \\
\hline PSNR & $\begin{array}{c}\text { Normalized } \\
\text { Cross } \\
\text { correlation }\end{array}$ & PSNR & $\begin{array}{c}\text { Normalized } \\
\text { Cross } \\
\text { correlation }\end{array}$ \\
\hline $\begin{array}{c}\text { Without } \\
\text { any effect }\end{array}$ & 31.1753 & 0.8585 & 5.5009 & 0.8583 \\
\hline Poission & 26.4138 & 0.8567 & 5.4884 & 0.8562 \\
\hline $\begin{array}{c}\text { Gaussian } \\
(0.04)\end{array}$ & 3.4559 & 0.8575 & 7.5361 & 0.8563 \\
\hline $\begin{array}{c}\text { Salt \& } \\
\text { pepper } \\
(0.04)\end{array}$ & 15.4707 & 0.8518 & 5.4653 & 0.8520 \\
\hline $\begin{array}{c}\text { Rotation } \\
(140)\end{array}$ & 2.0832 & 0.8585 & 8.2513 & 0.8583 \\
\hline $\begin{array}{c}\text { Scaling(0. } \\
6)\end{array}$ & 19.9849 & 0.8472 & 5.4094 & 0.8466 \\
\hline $\begin{array}{c}\text { JPEG } \\
\text { Compress } \\
\text { ion } \\
(40)\end{array}$ & 26.3845 & 0.7505 & 5.4564 & 0.7724 \\
\hline $\begin{array}{c}\text { Cropping } \\
\text { [20 20 } \\
250 \text { 250] }\end{array}$ & 5.0688 & 0.8484 & 4.0732 & 0.8231 \\
\hline
\end{tabular}

Table 4. The performance of the proposed watermarking method is evaluated for two different watermarks

\begin{tabular}{|c|c|c|}
\hline K=5 & $\begin{array}{c}\text { Skeleton.bmp } \\
(\mathbf{6 0} * \mathbf{6 0})\end{array}$ & $\begin{array}{c}\text { watermark.bmp } \\
\mathbf{( 5 0} * \mathbf{2 0})\end{array}$ \\
\hline Without any effect & 0.8585 & 0.9470 \\
\hline Poission & 0.8558 & 0.9457 \\
\hline Gaussian (nd=0.04) & 0.8575 & 0.9293 \\
\hline Salt \& pepper (nd=0.04) & 0.8518 & 0.9207 \\
\hline Rotation (140 $)$ & 0.8585 & 0.9470 \\
\hline Scaling $(0.6)$ & 0.8157 & 0.8902 \\
\hline Compression(q=40) & 0.7314 & 0.7539 \\
\hline Cropping[20 20 250 250] & 0.8484 & 0.9313 \\
\hline
\end{tabular}

\section{CONCLUSIONS}

The watermark can be embedded into n-level DWT decomposition. By the observations, when the Gain factor (K) value increases then distortion in the watermarked image increases and quality of the extracted watermark also increases.

This method is implemented using both bi-orthogonal wavelet (bior1.1) and orthogonal wavelet (Haar) transforms for observations. If we use $\mathrm{Bi}$-orthogonal wavelets for decomposition then distortion in the watermarked image is less compared with the Haar wavelet transformation. Hence, Bi-orthogonal wavelet transformation is effective for reconstruction of a watermarked image.
The algorithm is also verified with different color cover images (football.bmp and x-ray.bmp) of same size and the PSNR values are tabulated. Two different sizes of watermarks (60x60 and 50x20) are taken as inputs and the PSNR and Normalized cross correlation values are tabulated.

The performance of the proposed method is tested by applying different geometric and image processing attacks, such as salt and pepper noise, Gaussian noise, poission noise, JPEG compression, rotation, scaling and cropping attack. The proposed method, with stands for all these attacks at first and second levels wavelet decompositions.

\section{REFERENCES}

[1] A. M. Eskicioglu, J. Town and E. J. Delp, "Security of Digital Entertainment Content from Creation to Consumption," Signal Processing: Image Communication, Special Issue on Image Security, 18(4), pp. 237-262, April 2003.

[2] R. B. Wolfgang, C. I. Podilchuk, E. J. Delp, "Perceptual watermarks for digital images and video," in Proc. of the IEEE, Special Issue Identification and Protection of Multimedia Information, vol. 87, no. 7, pp. 1108-1126, July 1999.

[3] X. Xiang-Gen, C. G. Boncelet, G. R. Arce, "A multiresolution watermark for digital images," in Int. Conf. on Image Processing (ICIP'97), vol. 1, pp. 548-551, 1997.

[4] N. Kaewamnerd, K. R. Rao, "Wavelet based image adaptive watermarking scheme," IEE Electronics Letters, vol. 36, no. 4, pp. 312-313, Feb. 2000.

[5] I. Cox, J. Kilian, T. Leighton, and T. Shamoon, "Secure spread spectrum watermarking for images, audio and video," in Proc. IEEE Int. Conf. Image Processing, Lausanne, Switzerland, Sep. 16-19, 1996, vol. 111, pp. 243-246.

[6] K. Tanaka, Y. Nakamura, and K. Matsui, "Embedding secret information into a dithered multi-level image," in Proc. IEEE MILCOM Int. Conf., 1990, pp. 216-220.

[7] A. Piva, F. Bartolinin, V. Cappellini, and M. Barni, "Exploiting the cross-correlation of rgb-channels for robustwatermarking of color images," in Proc. Int. Conf. Image Processing, Kobe, Japan, Oct. 24-28, 1999, vol. 1, pp. 306-310.

[8] T. Tsui, X. Zhang, and D. Androutsos, "Colour image watermarking using multidimensional Fourier transforms," IEEE Transactions on Information Forensics and Security, vol. 3, no.1, pp. 16-28, Mar. 2008.

[9] N. Ahmidi and R. Safabakhsh, "A novel DCT-based approach for secure colour image watermarking," in Proc. Int. Conf. Information Technology: Coding and Computing, Apr. 2004, vol. 2, pp. 709-713.

[10] Roland Kwitt and Peter Meerwald and Andreas Uhl, "Colour-image watermarking using multivariate powerexponential distribution" Department of Computer Sciences University of Salzburg, A-5020 Salzburg, Austria. 